Voix et Images

\title{
III.1 Politiques éditoriales publiées en revue (BJ/NBJ)
}

Volume 10, numéro 2, hiver 1985

La barre du jour / La nouvelle barre du jour

URI : https://id.erudit.org/iderudit/200500ar

DOI : https://doi.org/10.7202/200500ar

Aller au sommaire du numéro

Éditeur(s)

Université du Québec à Montréal

ISSN

0318-9201 (imprimé)

1705-933X (numérique)

Découvrir la revue

Citer cet article

(1985). III.1 Politiques éditoriales publiées en revue (BJ/NBJ). Voix et Images, 10(2), 21-40. https://doi.org/10.7202/200500ar d'utilisation que vous pouvez consulter en ligne.

https://apropos.erudit.org/fr/usagers/politique-dutilisation/ 


\section{ANTHOLOGIE DES POLITIQUES ÉDITORIALES DE LA BJ ET DE LA NBJ}

\section{III.1 Politiques éditoriales publiées en revue (BJ/NBJ)}

Notes préliminaires

Les politiques éditoriales publiées en revue et retenues ici l'ont été selon les critères suivants:

- signées du nom de la revue;

- et/ou signées d'un des membres de l'équipe directrice;

- et/ou signées du ou de la responsable d'un numéro.

Il va sans dire, cependant, qu'on ne retrouvera pas ici tous les liminaires, présentations et avertissements de tous les numéros spéciaux, par exemple.

BJ, no 1, février 1965

\section{Présentation}

À l'heure actuelle le Québec se recrée dans une production littéraire tendue et inquiète. Â l'attitude révolutionnaire des jeunes poètes et romanciers nous ne pouvons que constater une solidarité et un dynamisme nouveau.

Nous ne saurions rester indifférents à ce mouvement. Nous tentons aujourd'hui de participer à ce dynamisme en présentant au public une revue littéraire à prix populaire. Celle-ci s'efforcera de mieux faire 
connaître nos écrivains d'hier et d'aujourd'hui. En plus des textes des collaborateurs, nous vous offrons la chronique "Les Inédits» qui saura sûrement vous intéresser. Notons que des études littéraires seront publiées dans les prochains numéros.

La Barre du Jour ne défendra aucune idéologie politique, mais elle ne pourra qu'acquiescer à tous les textes de valeur littéraire qui lui seront soumis, bien qu'ils fussent empreints de caractère politique. Car s'il n'y a pas de poésie engagée, il y a une poésie essentielle qui veut tirer l'image de l'homme vers la lumière et assurer à tous une place dans cette conscience culturelle qui s'éveille rapidement aux nécessités et par là se définit comme nécessité. D'ailleurs si nous pouvons maintenant nous prêter avec tant d'ardeur a la création de cette nouvelle revue, c'est en un sens dû à l'inévitable situation de lucidité dans laquelle nous plonge notre milieu. Nous voulons répondre à ce climat de lucidité et il nous semble que cette revue est le meilleur moyen d'atteindre notre but.

Les pages de notre revue sont ouvertes à toute collaboration littéraire. Le risque fragile de paraître hétéroclite sera compensé par la richesse qu'apporteront ces multiples collaborations. La Barre du Joưr s'efforcera d'être un port d'attache pour l'écrivain et le lecteur.

BJ, no 8, octobre-novembre 1966

\section{éditorial}

La Barre du Jour veut être dynamique, et ce à l'heure urgente où les écrivains québécois mettent fin à de vieilles spéculations sur la littérature, pour entrer enfin dans l'ère de son établissement. Pour cela, il nous faut s'engager et s'enrager totalement pour une littérature dont nous reconnaissons l'existence. Peut-être est-elle un peu fragile puisqu'à la fois elle existe et existe peu, mais elle est réelle tout de mème, et nécessaire surtout. Face au public, nous nous devons, d'une part, de promouvoir cette littérature et d'autre part, de la répandre à tous les niveaux. Ne pouvant par elle seule rénover entièrement de vieilles structures et les rendre plus propices à l'épanouissement de notre littérature, notre revue se doit de faire des revendications.

Or, La Barre du Jour ne peut soulever seule l'édifice de la "culture québécoise». Mais étant donné que cette littérature peut seule donner aux québécois leurs véritables racines et leur identité, nous croyons qu'il est nécessaire de former de nouveaux lecteurs. Et cela doit se faire par l'enseignement de cette littérature, dès le niveau secondaire. Nous revendiquons donc que notre littérature soit enseignée autant qu'une autre. Ici, nous n'entendons pas bannir l'étude des auteurs français, mais seulement les situer où ils se doivent 
d'être; c'est-à-dire situés en tant que représentants d'une littérature d'importation d'où nous ne pouvons tirer notre véritable identité.

Actuellement notre littérature est un sous-produit et elle le restera tant que l'enseignement la considérera comme telle. C'est l'école qui la première peut former un public - ce potentiel d'écoute que nécessite une littérature. La situation est urgente. Il nous faut de nouveaux manuels littéraires, mais il nous faut aussi aller plus loin. L'école normale qui forme les instituteurs, se doit d'enseigner notre littérature à ceux-là qui devront la transmettre et la faire connaître. Cela même leur est refusé, nous est refusé. Cette situation est inadmissible, surtout lorsqu'au niveau post-secondaire, il existe suffisamment d'ouvrages sur les lettres québécoises, pour donner une connaissance et une formation adéquate.

La Barre du Jour s'est donné une nouvelle équipe et elle entend intensifier ses exigences. En cela, elle consacre une grande importance à la publication de jeunes auteurs: c'est-à-dire tout ce qui est véritable création et recherche nouvelle. Cette nouvelle littérature ne s'assimile peut-être pas aussi facilement que la traditionnelle, à cause du degré de recul historique, mais il n'en demeure pas moins qu'elle permet par notre revue, d'ouvrir la porte sur l'avenir littéraire et cela autant pour le lecteur que pour l'écrivain. Notre revue peut aussi agir en redonnant vie et voix à de vieux auteurs qui sont demeurés sans tradition de lecture et donc sans vie littéraire à cause de la rareté des rééditions.

Nous ferons également tout en notre pouvoir pour que la véritable critique et l'essai vivent parallèlement à la création littéraire. Nous défiant de l'unanimité de la critique et des jugements portés sur certaines oeuvres, nous croyons que tout n'est pas dit d'une oeuvre lorsqu'une seule critique a été faite. Les études littéraires et les essais peuvent en se multipliant enrichir cette meme cuvre et la parachever, du moins la faire revivre au présent, puisqu'il est entendu qu'elle est une totalité, une aventure humaine à travers le temps.

M. St-P. I la barre du jour

- BJ, no 10, automne 1967

\section{d'une mise au}

Devant l'inquiétude ou l'indifférence que nous pourrions offrir aujourd'hui face à l'avenir des genres littéraires, il nous paraît inutile même de se demander ce à quoi correspondent de nos jours ces memes genres et les appellations qu'on leur donne, car qui pourrait établir des distinctions claires et acceptables par tous et partout entre le conte, le récit, la nouvelle et le roman ou encore entre poème, poème en prose et prose tout court, qui oserait s'étendre encore un peu plus sur ces 
distinctions anciennes qui ont perdu leur raison d'être, et pire encore, on ne pourra peut-être jamais s'entendre sur ce qui, ressortant de tous ces genres, s'appelle encore, d'un nom plus générique, la prose?

Un mot comme celui-ci qui caractérise une forme d'écriture, ne saurait recevoir de définition que s'il était comparé à ce qu'il n'est pas. Or, mis à part tout ce qui s'écrit en vers (bien que trop souvent de tels textes ne sont que l'effet d'une mauvaise disposition ou d'un étalement artificiel de la prose sur la page), toute écriture relève de la prose. De telle sorte qu'on ne peut définir la prose que par rapport au poème qui est un en vers du discours écrit, tandis que celle-ci en serait un en suite, l'endroit d'un discours qui va en droite ligne, un continu de l'écriture. Un tel mot, on le voit, défini par sa linéarité et pouvant servir à toutes fins et à toutes sauces ne pourrait cependant être utilisé consciemment sans qu'on lui trouve quelqu'air de fausseté. N'étant clair pour personne, on l'utilise abusivement quand, au fond, il est peut-être inutilisable? N'est pas prose qui veut, car rien n'est prose et toute écriture en est à la fois, mais dès qu'on détermine ainsi sa forme, on ne sait plus de quoi on parle exactement. D'un mot bien sûr, mais...

Un mot de trop peut-être, un mot ni ange ni bête pourtant, un mot qui de fait, ne correspond plus à un genre ni à aucune forme précise et auquel toutefois on tente encore d'en donner un ou une comme on donne parfois un sexe à un nom. Le nom d'une forme d'écriture si répandue et pourtant si informe qu'on ne sait plus à quoi elle s'applique, un mot encore qui ne veut rien dire, si ce n'est qu'autrefois il était intelligible et qui fut, pour un certain temps, le terme exact dont on se servait pour nommer tout ce qui n'était pas versification, un mot donc, qui ne rime à rien depuis que cette convention n'existe plus.

Encore un mot qui ne signifie rien et n'a aucun sens, un autre sans allure, devant l'avenir duquel on n'ose plus s'entendre et qu'il vaut mieux ici, laisser en suspens...

m. st-p. pour LA BARRE DU JOUR

- BJ, no 11-12-13, mai 1968

\section{Connaissance de Giguère (extraits)}

Les textes qui suivent $n$ 'ont pas pour but de mettre un point final ou meme de faire le point de nos connaissances sur cette ceuvre. Loin de résumer l'œuvre entière, ces études et ces témoignages ont pour seule intention d'entamer la recherche, d'amorcer cette connaissance. Ce livre constitue donc, par son ensemble, une approche de l'oeuvre, un défrichement et par là même, déjà une connaissance. 


\section{(..)}

Scruter cette oeuvre, la fouiller, l'analyser, en tâter le terrain et les «secrets» qu'il renferme, en reconnaître les repères les plus essentiels, parcourir les corridors de cette mine ou «la route qui mène à l'intérieur des terres»; telle est l'ambition des études et des témoignages qui suivent, melée au désir de mieux connaître ce personnage, quelque peu secret, qui écrivit un jour, non sans raisons, sir «JE SUIS LE MINISTRE DES AFFAIRES INTÉRIEURES», celles obscures, celles inextricables, et le jeu consiste à s'y perdre et s'y retrouver alternativement...

Jeu grave, s'il en est un, auquel Giguère vous convie et auquel, nous nous sommes laissés prendre.

m. st-p. / la barre du jour

BJ, no 15, aoat-septembre 1968

\section{Jean-Michel Valiquette}

Le plus étrange écrivain du Québec disparaît en ne laissant derrière lui que sa mort et quelques restes; fragments écrits en marge d'un thème unique. Poète de la négation absolue et de l'illisible, l'ironie du sort a voulu qu'il soit le premier et le dernier poète maudit du Québec.

(Marcel Saint-Pierre)

BJ, no 21, septembre-octobre 1969

\section{Saint-Denys Garneau (extrait)}

Le jour où l'angoisse qui habitait Saint-Denys Garneau s'est transformée chez d'autres en révolte avouée et vécue, un triste regard méprisant s'est abattu sur l'homme et sur ceux qui vécurent autour de lui cette ère de bêtise, d'ignorance et de totalitarisme. Triste, parce que l'histoire commandait leur attitude, méprisant, parce qu'ils avaient terni notre enfance, notre adolescence. Encore aujourd'hui certains d'entre eux font tache de graisse sur les aspirations saines et légitimes de notre collectivité. C'en était fini de la frustration et de l'impuissance. La parole et l'action allaient remplacer ces intolérables servitudes. Pour nous, Saint-Denys Garneau a toujours été un homme marqué, celui que l'on montre du doigt. ILS en avaient trop dit et trop fait pour un écrivain qui n'avait été que l'esclave et le reflet d'un désespoir qui - avec la révolution tranquille, la naissance de Parti 
pris, du M.L.F., etc. - était devenu à nos yeux pur masochisme. ILS avaient sanctifié l'obscure prison qui leur servait de maison et nous n'avions que faire de cette canonisaton. Au fond, ce qui sauve SaintDenys à nos yeux, c'est d'avoir été conscient; ce qui le perd, c'est de s'être laissé étouffer, de ne pas avoir trouvé en lui (écrivain) la matière et les arguments qui lui auraient permis de VIVRE, seul peut-être, mais en accord avec lui-même.

En 1951, Gilles Marcotte terminait un texte sur la poésie de Saint-Denys Garneau en écrivant: "Saint-Denys Garneau ne nous quitte pas». Et cela demeura vrai pendant trop longtemps. Aujourd'hui NOUS avons quitté Saint-Denys et sa place, nous la lui taillons dans l'histoire d'une littérature qui se faisait.

N.B.

BJ, no 26 , octobre 1970

\section{Mise au point}

«Et ainsi en va-t-il pour le mythe, pour le personnage dont on s'étonne, on s'amuse, on s'agace, dont on profite..."

Miron le magnifique Jacques Brault prétexte.

Le Colloque Miron a été un prétexte. Nous voulons assumer ce

Avec des moyens de bord, un temps limité, face à une situation d'urgence, des universitaires ont tenu à prendre position, à protester contre la loi sur les mesures de guerre. On est donc allé au plus pressant: enregistrer notre dissidence (face à cette loi), sans pour autant se faire d'illusions sur la portée de notre mode d'action.

Gaston Miron a été utilisé comme symbole.

Pour éviter tout malentendu, nous précisons que nous ne voulons pas, dans la situation actuelle, accorder plus de sympathie a Miron qu'à Gérald Godin et Michel Garneau. Ou encore qu'à M. Tremblay de la rue Sanguinet incarcéré pour le même délit d'opinions. Nous déplorons bien sâr ces emprisonnements, mais ceux-ci ne deviendront jamais plus arbitraires parce qu'il s'agit d'écrivains. La liberté d'expression doit exister pour tous, sinon elle est un leurre.

Il faut aussi souligner le fait que l'accession à la culture est un phénomène de promotion de classe, que la culture est un instrument de classe et qu'elle fait de son détenteur un privilégié. Nous sommes des privilégiés. Nous en sommes conscients. 
En publiant les textes du Colloque Miron, l'équipe de La Barre du Jour veut ainsi prendre position et marquer son opposition a la présente philosophie du pouvoir.

LA BARRE DU JOUR

\section{De notre écriture en sa résistance}

En 1963, nous avons lu Parti pris et nous avons compris. Il n'y avait pas d discuter: les positions critiques de cette revue ajoutées à notre expérience quotidienne du Québec - face aux premières ébauches d'une révolution qui de balbutiements en balbutiements devint tranquille - achevèrent de transformer notre impatience en un naturel contestataire.

Plus tard, les Éditions Parti pris publièrent Le Cassé (novembre 1964) et L'Afficheur hurle (janvier 1965). Les raisons qui poussèrent Renaud et Chamberland à écrire avec tant de désespoir et de violence ces livres nous apparurent légitimes et, de ce fait, inquiétantes: nous venions de comprendre ce qu'était notre lot. Miron, dont nous ne connaissions guère les écrits à cette époque, Miron, le déboussolé, le déraillé, l'aliéné, n'était plus seul. Du même coup nous devenions tous des colonisés, mais des colonisés conscients et, par le fait même, dangereux. Pourtant notre révolte ne nous apportait que des mots d'impuissance à prononcer, à écrire. Et nous avons choisi de ne pas les écrire.

1. L'esthétique psychologique traditionnelle ne tenait plus le coup devant l'esthétique suicidaire de Renaud, Chamberland et Miron.

2. Notre esthétique consisterait à préciser le défi littéraire d'une écriture désarticulée (nouveau joual syntaxique).

Ce qui fait la différence entre Miron et nous, c'est que nous avons compris collectivement que le système dans lequel nous existions avait fait de nous des êtres diminués. Cela nous l'avons appris dans la solidarité des premières discussions et des premières manifestations. Nous avons intellectualisé notre mal. Nous en avons compris le pourquoi et le comment. Tout ce cheminement que nous avons dû accomplir pour enfin connaître notre véritable identité, Miron l'a, contrairement à nous, vécu dans la solitude de sa chair.

«je suis malheureux plein ma carrure, je saccage la rage que je suis, l'amertume que je suis avec ce boeuf de douleurs qui souffle dans mes côtes. "” 
Lorsque Miron découvrira ses tares et son mal d'être Québécois, il écrira. Il parlera de lui et de son mal. Il vivra le je collectif aux risques et périls de son propre équilibre, ce je collectif qui fera de lui un Québécanthrope, un orignal bramant, un feu rouge alertant sur la place publique.

Parallèllement à la lutte écrite et orale que menait Miron, les jeunes intellectuels de l'époque, Jean-Marc Piotte, Pierre Maheu, Paul Chamberland et d'autres mettaient la hache dans les derniers arguments fédéralistes. Les vieilles images s'effritaient à un rythme effréné. Des mouvements s'organisaient. Bourgault et d'Allemagne au R.I.N., le M.L.P., le M.I.S., les manifestants, tous préparaient le combat. Les chansonniers répétaient à tous vents le pays (Mon pays), l'aliénation (Bozo les culottes), l'appartenance (Le grand six pieds), l'exploitation et la petite misère noire du Québécois. Pourtant celui-ci releva symboliquement la tête: il devint un grand six pieds et il construisit la Manic.

Miron poursuivait son enseignement, réintroduisant, chaque fois que cela était nécessaire, des arguments nouveaux aptes à faire progresser notre réflexion politique.

Alors que sur le terrain poético-politique tout semblait avoir été dit - et nous n'avions pas envie de devenir des répétiteurs impuissants - par ceux qui assumaient (par les tripes) l'angoisse et la révolte des Québécois, nous qui la partagions avons choisi de faire porter notre travail sur le langage. Pendant que d'autres renchérissaient sur notre situation alarmante, nous cherchions à combattre à l'intérieur de ses frontières une sémantique qui, à coup sûr, faisait le jeu culturel de ceux contre lesquels les «politiques» se battaient. $\grave{A}$ travers ces expériences, nous avons parlé de nous, de nos sexes si longtemps enfouis dans les neiges québécoises. Nous avons cherché et trouvé un décor urbain qui n'avait pas, ô miracle, la syphilis $2 .$. Nous avons rêvé, mais nos rêves précisaient notre volonté de vivre; ils traçaient les itinéraires futurs d'une attitude critique face à une littérature qui ne parvenait pas à se départir de ses mauvaises «habitudes». Nous acceptions mal que Marie-Claire Blais et Réjean Ducharme recommencent un jeu de fou (la valse des vieilles rengaines). Nous avons refusé les big mamelles remplies de lait frais qui de Québec jusqu'à Montréal se frayaient un chemin à travers les étoiles, les sables mous, les partances, etc. Nous avons refusé de faire survivre le passé. Nous avons préféré le comprendre et saluer ceux qui annonçaient déjà les temps de la liberté (cf. numéros Giguère et Automatistes).

Nous avons voulu que trébuche sur nos textes la critique suffisante. Nous avons refusé de faire plaisir à ceux qui misent sur la facilité de quelques mots clés-succès.

Nous avons volontairement piégé le langage afin que la culture bourgeoise ne se serve pas de nos ouvres pour perpétuer ses valeurs. 
Nous avons refusé de faire plaisir à ceux qui comptent sur l'inconscience et qui voudraient voir cette dernière transmise de génératon en génération par des écrivains dociles.

Tant et aussi longtemps que l'establishment fera la pluie et le beau temps avec le travail et la santé des salariés québécois, tant et aussi longtemps que des hommes seront emprisonnés pour leurs idées, nous aurons besoin d'une littérature:

1. subversive susceptible de changer les valeurs reçues.

2. opérante efficace: n'hésitant pas à supprimer images et mémoires quand elles seront sur le point d'être récupérées. Bref, conséquente et corrosive.

3. délictueuse

dans sa grammaire, inopérante pour qui pense le présent comme le passé.

Sur Miron, ajoutons ceci: il est un des rares poètes à ne pas avoir fait dévier sa revendication politique sur le plan culturel (cf. Duguay, Chamberland, etc.). Pendant que d'autres vivent déjà dans l'acquis d'une nouvelle réalité nationale (ils en vivent, ils en meurent), Miron continue de lancer son appel. Et s'il demeure aujourd'hui un des poètes les plus écoutés, c'est que le Québec a plus que jamais besoin de sa liberté.

Nicole BROSSARD

Roger SOUBLIËRE

Ce texte a suscité de vives discussion à La Barre du Jour. Faute d'unanimité, il n'engage que ses signataires.

1. La braise et l'humus in L'Homme rapaille, P. 53.

2. Aujourd'hui le mal des villes québécoises porte le nom d'anglicisation galopante.

BJ, no 29, été 1971

Notes sur une pratique (extrait)

\section{Déconstruire cette poésie}

Donc, une nouvelle écriture (écriture matérialiste, i.e. considérant les phénomènes comme liés entre eux (langue / histoire), pro- 
duisant et transformant) va avoir à dénoncer cette idéologie du textemiroir, pour ensuite élaborer une théorie qui va situer l'écriture et ses implicatins politiques et idéologiques, permettre une véritable pratique de classe. Lutte du matérialisme contre l'idéalisme, notre travail est basé sur le concept de "matière», à savoir une "catégorie philosophique servant à désigner la réalité objective donnée à l'homme dans des sensations qui la copient, la photographient, la reflètent, et qui existe indépendamment des sensations. $1_{\gg}$ Travail qui consiste à énoncer ce rapport dialectique matière / pensée en pervertissant le texte. MONTRER QUE LES CHOSES EXISTENT HORS DE NOUS. Mimer la représentation poétique et la détruire en accordant la primauté àla matérialité des mots (production du signifiant). Nier à l'écriture sa véracité a priori, l'exposer comme lieu de «taxation» d'un sens. Écrire non plus pour un «lecteur», mais pour une «lecture» qui désaxe le phénomène habituel: l'identification au texte. Attaquer en somme un tabou social: le langage.

Qu'en reste-t-il avec la communication? Terme éminemment subjectif et idéalisé, il est remplacé par celui de «rapport» entre un constant idéologique (la poésie) et celui qui s'apprête à le subvertir dans les pages qui vont suivre.

François CHARRON Roger DES ROCHES

1. V. Lénine, Materialisme et empiriocritisme, éd. du Progrès, URSS, p. 169.

BJ, no 29, été 1971

\section{Les inédits (extraits)}

Claude Gauvreau a décidé d'en finir. De régler ses comptes avec l'absurde. D'aller voir ailleurs s'il y est. Il a pris le chemin le plus court. Moniste athée, il n'avait de conseil à demander à personne.

Je refuse de chanter les louanges de Gauvreau. On ne vante pas les mérites d'un anarchiste libertaire. Ses écrits devraient suffire à l'enraciner dans notre mémoire et à lui tailler la place qui lui revient dans notre histoire littéraire.

Et pourtant la foi et l'espoir finissent toujours par s'estomper. Alors rien ne va plus. La mort de Gauvreau s'inscrit comme une suite logique à un refus global... 
BJ, no 31-32, hiver 1972

\section{Tiret}

Où en sont rendus les intellectuels québécois? Entre autres ceux qui ont été à l'origine des analyses socio-politiques les plus essentielles au projet d'existence nationale du peuple québécois et de son émancipation. Ce numéro spécial sur Parti pris tente de répondre à la question en faisant le point sur cinq ans d'analyse et de recherche, sur les trois années qui ont suivi et qui apparaissent comme un temps de mise en pratique quotidienne, clandestine et/ou publique de l'effet didactique de la revue Parti pris.

Pour plusieurs intellectuels, militants et citoyens, Parti pris a été à l'origine de la longue explication, pour une fois rationnelle et conséquente, de l'aliénation québécoise, voire des structures sociopolitiques discriminatoires et des phénomènes d'exploitation en contexte canadian.

Parti pris a été promoteur d'un vocabulaire qui a d'abord servi à l'analyse pour ensuite être transformé en générateur d'action. Nous en sommes, semble-t-il à cette dernière phase. L'élaboration des stratégies prime donc maintenant davantage sur le renouvellement théorique et analytique de la situation québécoise. D'où l'emploi d'une terminologie de plus en plus réductrice des problèmes québécois, d'où une analyse quotidienne et forcément morcelée des faits et événements sociaux du Québec, d'oủ le sens anémié d'une large part du vocabulaire révolutionnaire né de l'expérience Parti pris. L'intellectuel québécois n'a pas renouvelé son vocabulaire - quand je dis renouveler, je ne signifie pas là un emprunt systématique au lexique utilisé dans l'analyse marxiste - - . Bien que la multitude de ses activités politiques lui donne un sentiment de vitalité, il vit présentement (sur le plan de la pensée politique) une phase d'attentisme qui, pour autant qu'elle est liée à l'arrivée de l'EVENEMENT, risque de se prolonger trop longtemps. Ce qui aura pour effet de réduire, d'année en année, une problématique en une pléiade de bon voeux.

Parti pris disparú, Socialisme québécois se faisait rarissime, nous passons graduellement du vocabulaire inspirateur de Parti pris aux vocables «sloganesques» de l'hebdomadaire, vocables qui d'une part sont inhibiteurs de réflexion (nous sommes déjà au courant) mais qui, d'autre part, sont générateurs d'action et de participation. La question est de savoir si nous voulons embrigader ou expliquer et convaincre. (Mais avons-nous le choix de l'urgence de choisir?). On dira que l'un n'empêche pas l'autre. Oui. Car on ne peut utiliser parallèlement deux lexiques socio-politiques sans que l'un des deux ne reste en retrait - surtout quand ils se recoupent dans leurs fins. 1 
Encore faudrait-il pour le moment que l'on retrouve l'inspiration qui a présidé à l'avènement de Parti pris afin d'imaginer un nouveau moyen d'action textuel, pour ainsi dire inexistant sur le plan théorique depuis la disparition de la revue.

Parti pris nous a permis d'entrevoir l'image d'une société québécoise autonome et de travailler à sa réalisation. Depuis, nous constatons - et c'est là le paradoxe - qu'alors même que les années d'existence du Québec sont comptées (un folklorique, very enchanting coin québecois dans un vaste Canada n'a rien d'impensable), nous assistons à une profusion de manifestations de tout ordre qui témoignent de la gestation d'une véritable culture québécoise. Ce paradoxe que nous vivons quotidiennement tient-il du mirage alors que nous sommes à courir nos derniers milles ou annonce-t-il l'apparition d'un pays nouveau - qui ne soit pas une république de sapins (s'il en reste!) - sur l'échiquier international?

Nicole BROSSARD

1. Il ne faudrait surtout pas voir dans l'usage de plus en plus fréquent du terme uclasse laborieuse» en remplacement du terme «peuple québécois» une illustration de ce que j'appelle un vocabulaire renouvelé. Dans cet exemple, les termes ne s'opposent pas comme c'était le cas avec «canadien-français» et "québécois». "Classe laborieuse» ne sert ici qu'a faire réapparaître la réalité sociale du Québec dans l'ordre des priorités tactiques devant entraîner l'indépendance et/ou le socialisme. Entre autres faut-il ajouter qu'entre l'euphémisme "classes défavorisées" et l'aseptique appellation "classe laborieuse», il existe une réalité lexicale apte à servir plus qu'une analyse événementielle et qui permettrait peut-être d'aborder la deuxième étape du questionnement de la problématique québécoise.

\section{À l'état de fiction}

... ils se sont transformés en étranges versificateurs et qui, diraiton, dans une syntaxe fort convenable et prétexte seulement, bien entendu. Car les textes contes, la trans-version poétique que nous vérifions ici en publiant l'effet de Babel insoupçonné, n'annoncent rien, ne présagent de rien sinon d'un effet. Quand les mots deviennent générateurs à la fois de leur propre fiction mais aussi d'un double sens continu qui n'appelle pourtant que la seule interprétation des textes tels qu'en eux-mêmes présentés à la lecture, les mots n'insistent vraiment que pour dire qu'à cela ne tienne...

le cela des textes s'infiltre car il est bien question de lecture et d'œil inquisiteur. À ceci qui les juge, il n'est de proposition que l'indice et le jeu de pistes qui le complète mais insidieusement comment? 
il n'est pas ici question d'inventer de quelconques ancêtres québécois qui, $d$ 'ailleurs, auraient vite fait de renier leur progéniture. Les nouveaux fabriquants de contes, c'est bien certain, retrouvent un fond de mémoire à leur chaque texte écrit au pied de la lettre. Le jeu s'installe, déà embrouillé confond les esprits pollués par les lectures faites et entretenues dans LE GROS BON SENS littéraire.

il est connu, sinon détecté et analysé que cette littérature du gros bon sens dont nous avons à faire que de nous en défaire, cherche à gauche et à droite des complicités et qu'elle sera tenté de voir dans des textes-contes rédigés en bonnes et dues formes grammaticale et syntaxique, une image faite à la ressemblance de sa plénitude de sens et de sentence (au sens où elle dicte par décret culturel un contenu idéologique qui, sous prétexte d'aller de soi, fraude irrémédiablement l'exercice de lecture).

ici rien ne va de soi. Les efforts supplémentaires engageants sont à la fois du ressort de l'écrivain et du lecteur. A celui donc qui s'avise.

N.B.

— BJ, no 39-40-41, printemps-été 1973

\section{(Onze analyses)}

\section{Vague de précision}

Arriverons-nous à démontrer quoi que ce soit? Ainsi en est-il de la question que nous suggérons au lecteur, un parmi tant d'autres à se dévoyer de poésie, celle d'avant l'étape décisive que nous frôlons collectivement dans les termes d'une agitation "passive».

Et bien entendu, s'il s'agit d'une «critique», c'est qu'elle franchit l'étape de la surveillance morale et se dispose à sommeiller dans le texte sans avertissement autre et but que d'en tirer les ficelles.

Ainsi fut-il admis qu'une lecture décapitante (dont on omettrait volontiers la tête thématique) obligerait de "délire» méthodique. Et cela se fit à froid, à l'ombre des matières chaudes qu'il fallut stimuler, leurs structures n'apparaissant qu'à bout de lecture et de mise en place, sur chaque plan (phonique, sémantique, syntaxique, graphique) attestant l'ordre caché des équivalences formelles.

Le temps n'est pas à l'oubli de ce qui fut signifié mais il est aussi venu de se demander dans quelle matière ce jeu poético-socio-culturel fut incarné, lors même que nous causerions l'effet d'une préface, d'une rature, à toutes ces lectures antérieures qui furent bien débordantes (le trop plein d'illusions sémantiques les ayant bousculées dans un présent politique qui les fait maintenant s'éteindre) d'entrain. 
C'est qu'en effet, notre acte de lecture indisposera parce que s'y jouant à vide; puisqu'il ne sera question ici que d'y voir clair sans pour autant que nous en tirions maintenant les conséquences. À notre projet donc, point de conclusion sinon les vôtres.

Nous avons indiqué les lieux de l'incision, les lignes essentielles sur lesquelles il faudra appliquer de tout le poids du stylet pour pénétrer «le dedans» des textes que nous suggérons de lire comme un d'abord. Ensuite vous verrez.

N.B./LA BARRE DU JOUR

BJ, no 50, hiver 1975

\section{(Femme et Langage)}

\section{Préliminaires (extraits)}

Pour moi un travail immense est à faire sur la question de la relation de la femme québécoise à l'histoire (ce matriarcat de la survivance), à notre fiction collective et à notre propension seculaire à fonctionner au niveau du mythe 1 ... De plus, ce travail, en termes littéraires, il appartient à la femme de l'amorcer, dans la mesure où elle seule peut tenter l'exploration du désir féminin, de l'espace féminin, de la pratique de femme dans le champ brouillé des pouvoirs phallocratiques. Elle seule peut actuellement poser des questions aux textes d'une première société de mâles assumant leurs désirs, leur sujet, dans un érotisme condescendant quelqu'en soit les a priori "révolutionnaires». Elle seule peut formuler pour elle d'abord, pour les autres ensuite, le sujet féminin et ce faisant, faire éclater nos pratiques d'écriture.

C'est ce qui peut expliquer en un sens pourquoi nous avons écarté l'éventualité, dans ce numéro, de toute participation masculine. Et ce serait d'être bien mal avisé que de voir ce numéro de femmes comme une chronique, un ghetto de la maladie de femme.

$$
* * *
$$

Ce numéro, il était dâ et peut-être en même temps prématuré. $\mathrm{Da}$, en ce sens qu'il vient à point pour créer les ouvertures de questionnement qui s'impose à quelques femmes d'ici qui ont tous les jours à jouer du langage dans l'exercice de leur profession. Prématuré, dans la mesure où sur 16 femmes invitées à participer à ce numéro seulement 9 ont accepté et réalisé un texte. D'autres avaient accepté, mais pour des raisons de difficultés personnelles se sont arrêtées en cours de rédaction. 
La question-hypothèse qui fut à l'origine formulée était la suivante: comment la femme qui utilise quotidiennement les mots (comédienne, journaliste, écrivain, professeur) peut-elle utiliser un langage qui, phallocratique, joue au départ contre elle? C'était en fait poser la question à celles qui écrivent en leur demandant si, femmes, elles avaient, pouvaient avoir, pensaient avoir au langage, un autre type de relation que celle établie et pratiquée par les hommes.

En fait, la plupart des textes ne répondent pas directement à l'hypothèse de travail mais ils réussissent à faire état de lieux verbaux interdits, de contre-sens culturels et idéologiques, de la fiction, du corps de la femme, etc.

Ce numéro n'a pas l'homogénéité à laquelle je m'attendais (mais au fond, cela importe peu - même si...). Avant tout, il fallait tenter l'expérience du miroir, de l'interrogation. Tenter la femme à son propre jeu de maux.

Nicole BROSSARD

1. «Et tout comme l'idéologie bourgeoise se définit par la défection du nom bourgeois, le mythe est constitué par la déperdition de la qualité historique des choses: les choses perdent en lui le souvenir de leur fabrication.» Roland Barthes in Mythologies.

BJ, no 56-57, mai-aout 1977

\section{(Le corps les mots l'imaginaire)}

Donner à lire des écritures de femmes. Telles que nous sommes dans le sujet de nos textes, ce même sujet sans doute, Le corps les mots l'imaginaire qui nous traverse, isole et regroupe dans les multiples possibles de la vie souterraine/la réalité.

C'est bien en réalité que mises en situation d'écrire, posant le geste d'écrire comme un acte telles nous pouvons intervenir dans cet espace réservé aux mots. Ça prend alors une autre dimension.

n.b.

NBJ, no 58, septembre 1977

\section{Liminaire}

Existe-t-il une fonction précise de la revue dans un contexte littéraire? Certes mais les contextes varient. Et on peut aussi penser que chaque revue trouve sa fonction spécifique en vertu des divergences et des différences qui tôt ou tard finissent par surgir dans le milieu ou au milieu des questions politiques auxquelles sont maintenant soumises toutes les écritures. 
La création d'une nouvelle revue indique, dans la plupart des cas, une insatisfaction par rapport aux revues déjà existantes. C'est ce qui s'était produit lors de la fondation de La Barre du Jour en 1965. Depuis nous avons publié cinquante-sept numéros.

La Nouvelle Barre du Jour: par là, nous voulons indiquer un désir de renouveau lié à cette insatisfaction à l'égard des revues existantes, y compris La Barre $d u$ Jour. Mais plus encore, ce qui a suscité notre intention de recommencer, c'est un ensemble de circonstances subjectives et objectives qui nous amènent à vouloir un lieu-carrefour de l'écriture et de la pensée d'ici. Il s'agit pour nous de donner à lire des textes qui puissent à nouveau rendre effervescente et stimulante une littérature qui, pour le moment, apparaît hésitante.

La parution de chaque numéro d'une revue devrait en soi être un événement qui nourrisse la production individuelle des écrivains. Mais pour cela, il faut s'écrire régulièrement et qu'une continuité s'installe dans le rapport que nous entretenons avec les textes produits par les autres. D'où pour nous l'importance de Histoire d'écrire, «sentier de la création" où des écrivains disent leur rapport à l'écriture et le comment de leurs textes; un lieu d'essai permettant hypothèses et synthèses sur des sujets qui nous touchent de près ou de loin dans nos pratiques de vie et d'écriture; des Commentaires, des réflexions critiques sur des livres d'ici ou d'ailleurs, récents ou pas, mais pouvant servir de tremplin à l'écriture, à l'imaginaire et à la pensée. Le pourquoi et le comment des formes contenues ainsi que leurs effets.

Conséquemment, il nous apparaît important de synchroniser l'écriture et les courants de pensée les plus dynamiques, qui élargissent sans cesse nos champs d'action, de réflexion et de bien-être.

D'une génération à une autre, ce qui nous intéresse, c'est la démarche de tout sujet-conscience donnant signe de vie dans une forme incitative.

NBJ, no 92-93, juin 1980

\section{La Poésie en 1980 (extrait)}

Plusieurs signes avant-coureurs permettaient de prévoir un retour en force du je, du lyrisme, du lisible, d'un vocabulaire que certains n'avaient pourtant, malgré les discours dominants qui se sont succédés depuis dix ans, volontiers terroristes, jamais abandonnés au cours de ces années. Si besoin en était, cette anthologie en fournirait la preuve; qu'il suffise de relire les textes antérieurs de la plupart des auteurs qui y sont représentés pour mesurer le chemin parcouru. La 
poésie des années 80 sera plus riche de différences qu'elle ne l'a jamais été, mais elle s'inscrira dans la continuité.

michel beaulieu/la $n b j$

NBJ, no 100-101, mars 1981

\section{Liminaire (extraits)}

Revenir, une fois par année par exemple, revenir entièrement à la poésie. Au lieu d'où nous parlons - écrivons.

LA NOUVELLE BARRE DU JOUR n'est pas exclusivement une revue de poésie. Mais la plupart des textes qui y circulent, outre les poèmes proprement dits, essais, commentaires, proses diverses, sont marqués au coin du langage et de la pensée poétiques.

L'édition québécoise connaît des périodes difficiles de plus en plus longues (et répétées). Le recueil de poèmes, s'il lui arrive d'être publié, est lu avec des retards plus ou moins considérables. Ainsi se perd de vue le moment précis où le poème aurait dú s'inscrire. C'est cette sorte de retards que le présent numéro voudrait contribuer, entre autres, à combler.

On ne trouvera pas ici, loin de là, faut-il le préciser, toute la poésie québécoise. On lira des fragments de ces écritures que la NBJ entend lire et faire lire. La revue demeure un lieu de travail. Près de la langue, ce palais du poème.

D'année en année, poursuivre, malgré tout, la poésie.

NBJ, no 102, avril 1981

\section{(La Femme et la Ville)}

\section{Pré(e)}

La ville fascine jusqu'à l'étourdissement. Réservoir inépuisable pour l'imagination, elle crée ses propres mythes de blasphèmes et de séduction. Elle soutient toutes les comparaisons s'offrant comme un éventail de sensations qui oscillent entre l'horreur et l'utopie, entre l'exaltation et la désintégration. La ville au crépuscule est un reflet de la cité, la polis des hommes. La ville, via l'image, via les corps comme un calcul mental. 
La ville fabrique sans cesse des textes: de lois, de fiction. Ici treize femmes circulent dans la ville en encre et en pensée. La ville patriarcale sans relâche, patriarcale jusqu'aux dents. Plus on s'approche de son centre, le down town, plus violente et réitérée se fait la propagande patriarcale comme un poing, un néon, un trottoir dur. Les visages s'effacent, restent les habits, en catastrophe.

Que vienne le temps des monstresses, des anges et des vénézuelles. L'eau, le vertige, le noeud: les manuscrits sont à ce prix.

Loger la ville dans son oeil, comme en vue: une cible visionnaire.

Nicole Brossard

NBJ, no 105, septembre 1981

\section{Liminaire (extrait)}

\section{... de suite}

Une nouvelle équipe prend la relève. Question de préciser ici que la NBJ poursuivra son travail d'ouverture en tant que lieu de créations et de recherches. Question de réaffirmer la trace que, depuis plus de quinze ans (de la Barre du Jour à la dite Nouvelle), elle s'efforce de marquer dans l'inédit ou l'autrement dit. Question aussi de rappeler qu'une orientation ne se crée pas d'office et qu'elle dépend avant tout des auteurs et des collaborateurs qui la font. Peut-être bien qu'a l'instant de commencer son activité éditoriale, la nouvelle équipe tient à en appeler tant aux auteur(e)s qu'aux lecteurs ou lectrices pour que continue à se jouer le dynamisme essentiel à toute entreprise de ce genre.

Le collectif de direction

(Hugues Corriveau, Louise Cotnoir, Lise Guèvremont)

NBJ, no 118-119, novembre 1982

\section{(Traces écriture de Nicole Brossard)}

\section{Liminaire}

Depuis 1965, des textes s'avancent «Sur fil de lumière» pour dire un travail attentif à toutes les manifestations du changement culturel, social. La place de l'écriture de Nicole Brossard dans la littérature québécoise actuelle en est une de constante poursuite du renouvel- 
lement de la forme et du sens. Des débats neufs ont souvent pris source dans ces œuvres: le texte, le formalisme, la transgression, le féminisme, le lesbianisme, la modernité. Une passion d'écriture traverse ce corpus alimenté au monde des idées, de l'émotion et du corps. Une passion d'écriture qui parle dans les mots revisités avec lucidité. La nouvelle écriture trouve là un cheminement fait d'affirmations et d'hypothèses, de tensions et de rigueurs. S'interroger sur cette production déjà abondante mais toujours en élaboration ce n'est pas figer l'œuvre dans des approches analytiques qui viendraient la rendre lettre morte, c'est il me semble au contraire souligner, remarquer ce qui dans cette ouvre réactive sans cesse le goût d'analyser, de lire et d'écrire. Dans cette optique, le projet d'un colloque sur une production récente et en chantier vient tout simplement montrer le comment d'un travail à travers la lecture de textes qui sont là concrets à écrire ce qui se trame dans le langage quand il se fait exploration, étreinte, pensée, plaisir, questionnement. L'écriture de Nicole Brossard a laissé manifestement des traces dans la littérature québécoise, des textes sont réunis ici qui en témoignent en précisant l'impact, en inventoriant les lignes. L'ouvre demeure un livre à déchiffrer, approchons-nous d'elle.

Claude Beausoleil septembre 1982

NBJ, no 124, mars 1983

\section{(Femmes)}

\section{Liminaire}

Nous avions demandé à trois auteures, soit Carole David, Denise Desautels et Renée-Berthe Drapeau, de solliciter des textes auprès d'autres auteures peu ou pas encore publiées par la revue. Voici donc ici rassemblées celles qui ont écrit ce numéro particulier, chaque année réservé aux écrivaines qui cette fois tiennent le pari de n'avoir de thème autre que celui de leur propre venue et de leur propre écriture.

Le collectif de direction

NBJ, no 140, juin 1984

\section{Sortie/exit (extraits)}

Notre passage à la direction de la revue aura été marqué, nous semble-t-il, par une ouverture de plus en plus grande aux auteur(e)s. Ce sont eux et elles qui, par la confiance qu'ils ou elles nous auront 
témoignée, auront le mieux réalisé le projet de cette revue littéraire, soit d'en concevoir l'existence comme un lieu ouvert où s'articule la vitalité même du travail d'écriture. Ce sont eux et elles qui auront saisi cette chance d'avoir sous la main un tel instrument.

(...)

Toutes ces réalisations laissent présager de l'avenir et prouvent que cette revue, à la veille de fêter son vingtième anniversaire de fondation (à partir de La Barre du Jour), continue de travailler pour l'actuel et se préserve de l'usure. Faudrait-il, pour s'en convaincre, ajouter à la liste déjà donnée les noms des nouveaux et nouvelles auteur(e)s qui ont cru en La Nouvelle Barre du Jour en y publiant, nombreux et nombreuses, leur premier texte, faudrait-il nommer tous ceux et toutes celles qui, déjà plus connu(e)s, continuent d'enrichir cette revue en lui confiant leur travail permanent?

Hugues Corriveau Louise Cotnoir Lise Guèvremont 